\title{
Comparison of Saccular Function in Nonmusicians and Violinists Using cVEMP: A Pilot Study
}

\author{
Eva Eadle D’Souza ${ }^{1}$ Krishna Yerraguntla ${ }^{1}$ \\ ${ }^{1}$ Department of Speech and Hearing, Manipal College of Health \\ Professions, Manipal Academy of Higher Education, Manipal, \\ Karnataka, India
}

\begin{abstract}
Address for correspondence Krishna Yerraguntla, PhD (Sp\&Hg), CCC-A, Department of Speech and Hearing, MCHP, MAHE, Manipal 576104, Karnataka, India (e-mail: krishna.y@manipal.edu).
\end{abstract}

\begin{abstract}
Keywords

- cervical vestibular evoked myogenic potentials (cVEMP)

- early identification

- music

- noise-induced hearing loss (NIHL)

- sacculocollic pathway

- violin

Introduction Human auditory and vestibular systems change due to noise exposure. Professional musicians are often subjected to loud music and longer durations as part of their practice. Although the effects of music have been explored extensively on the auditory system, it is important to understand changes in the vestibular system also. The current study is aimed to compare cervical vestibular evoked myogenic potential (cVEMP) findings in nonmusicians and violinists to understand if there are any changes in the P1 and $\mathrm{N} 1$ latencies and absolute amplitudes in the violinists' groups because of their exposure to violin music.

Materials and Methods Twelve participants (6 nonmusicians and 6 violinists) of both genders were included in the study. Pure tone audiometry and distortion product otoacoustic emissions (DPOAEs) were performed on all the participants. CVEMP P1 and $\mathrm{N} 1$ latencies and absolute amplitudes were obtained, and overall mean differences were compared within and between groups.

Results Pure tone average and DPOAE were within the normal range between and within the groups. Results indicate that P1 and N1 absolute amplitudes and latencies were slightly prolonged in the violinists' group; however, the mean difference was not statistically significant. Comparison of mean absolute amplitudes and latencies between the ears in the violinists' group showed longer latencies and greater absolute amplitudes in the left ear of violinists as compared with the right ear. In the study, the violinists' group consisted of participants who had an average daily exposure of about one-and-a-half hours and had an experience of playing the instrument for more than 5 years.

Conclusion CVEMP is useful in detecting early changes in the saccule that may occur due to noise exposure. It can be concluded that, even before a clinically detectable hearing loss or vestibular damage, changes in saccule are observed with the help of CVEMP and should be included in the audiovestibular test for early identification.
\end{abstract}

\section{Introduction}

Music is a recreational as well as a professional activity that has been practiced throughout the years. Although music is deemed to be a fun-loving activity, it is proven to have detrimental effects on an individual's hearing. ${ }^{1}$ Musical information is comprised of a more balanced and meaningful arrangement of musical information, while on the contrary, noise is often less constrained. ${ }^{2}$
Published online September 9, 2021
DOI https://doi.org/ $10.1055 / \mathrm{s}-0041-1735384$ ISSN 2581-9607
(C) 2021. Indian Society of Otology.

This is an open access article published by Thieme under the terms of the Creative Commons Attribution-NonDerivative-NonCommercial-License, permitting copying and reproduction so long as the original work is given appropriate credit. Contents may not be used for commercial purposes, or adapted, remixed, transformed or built upon. (https://creativecommons.org/licenses/by-nc-nd/4.0/). Thieme Medical and Scientific Publishers Pvt. Ltd. A-12, 2nd Floor, Sector 2, Noida-201301 UP, India 
The human auditory system undergoes physiological changes due to noise exposure, specifically outer hair cells (OHCs) causing permanent or temporary damage to the structures and functionally, resulting in decreased auditory sensitivity. ${ }^{3}$ This type of noise exposure typically results in damage at higher frequencies, commonly affecting the region between 3 and $4 \mathrm{kHz}{ }^{4}$ To regulate these effects, Occupational Safety and Health Administration has set the permissible sound pressure level at 85 decibel A (dBA) for an exposure duration of 8 hours. A similarity in the pattern of damage in the cochlea and the vestibule was observed after intense exposure to noise. ${ }^{5}$ Reduced hearing in both ears, ringing sensation in the ear, inability to hear a speaker in background noise, and dizziness are some of the most commonly reported symptoms of noise-induced hearing loss. ${ }^{6,7}$ The onset of vestibular symptoms has been seen without the evidence of measurable hearing loss in persons exposed to industrial equipment noise. ${ }^{8}$ Illustrative human studies have reported a greater percentage of complaints related to the vestibular system, ${ }^{9,10}$ and increased cervical vestibular evoked myogenic potential (cVEMP) latencies in individuals who experienced a high level of noise exposure. Professional musicians are subjected to a high amount of practice hours and are at high risk of hearing loss that can be attributed to long-term exposure either due to playing musical instruments or the use of personal music systems. ${ }^{11}$

A study conducted on individuals using personal music systems for more than 1 hour, every day for 2 years, reported significantly prolonged absolute peak latencies in cVEMP with latencies of P13 and N23 peaks also being prolonged. ${ }^{11}$ This study demonstrates the unfavorable consequences of personal music system use on the sacculocollic pathway and the results obtained were similar to that of occupational noise exposure. Reduced vestibular excitability is observed by prolonged P13 and N23 latencies among musicians along with the other audiological findings. ${ }^{12}$

The effects of occupational hearing loss have been studied in musicians and predominantly have the same characteristics as that of noise-induced hearing loss, but variations may exist in symmetry when instruments such as the violin are taken into consideration. The music conveyed by the violin maybe classical or western and usually extends in the lower frequency ranges from 196 to $659.3 \mathrm{~Hz}$. It must be noted that the sound pressure level measured from the violin was approximately $85.4 \mathrm{~dB}(\mathrm{~A}) \cdot{ }^{13}$ Violists and violinists had poorer thresholds at 3 to $6 \mathrm{kHz}$ in the left ear as compared with the right ear, consistent with the left ear's greater exposure from their instruments. ${ }^{14}$

Recent research is inclined toward illustrating the influence of the use of musical instruments on the vestibule. In the case of violinists, it is important to understand whether the effect of exposure to music may influence an individual's vestibular responses. The auditory and the vestibular system have common genealogy and anatomical proximity, and the vestibular end organs can adversely be affected by the sound produced by the violin. The proximity of the violin to the ear and posture utilized while playing the violin as it involves tension in the sternocleidomastoid muscle that is innervated by the inferior vestibular nerve could affect the human auditory and balance system. Effects on the auditory system have been studied, but its effects on the balance system have not been established. Hence, there is a need to study the effect of sound produced by violin on the saccular system.

\section{Methodology}

\section{Participants}

Twelve student participants, from the age group of 18 to 30 years, after informed consent, volunteered to be a part of the study. A case-control study design was utilized. Control group and the experimental group comprised nonmusicians and violinist, respectively. Based on the inclusion and exclusion criteria, age-matched six participants were recruited in each group. With the approvals from the Institutional Ethics Committee, Kasturba Hospitals, Manipal (IEC:136/2020) and CTRI Registration, data were collected at the Department of Speech and Hearing, Manipal College of Health Professions (MCHP), Manipal Academy of Higher Education (MAHE), Manipal, Karnataka.

The violinists' group consisted of participants who had an average daily exposure of about one and a half hours and had an experience of playing the instrument for $>5$ years. Furthermore, they had no middle ear pathologies or any neurological and psychological conditions. The nonmusicians group had no experience of playing any instruments, both professionally and for recreational purposes. Similar criteria as that of the experimental group were utilized to exclude participants from the control group. All participants were advised to avoid the use of muscle relaxant, ototoxic or vestibulotoxic drugs for 48 hours before testing. Participants who for any reason could not withdraw these drugs were not considered for the study. The recruited participant first underwent an audiological evaluation, which included pure tone audiometry, screening tympanometry, and distortion product otoacoustic emissions (DPOAE) (to ensure that participant fit the inclusion criteria), followed by a vestibular evaluation assessing the latencies and absolute amplitudes of P13 and N23 peaks with cVEMP.

\section{Instrumentation and Procedure}

A calibrated pseudo-two-channel audiometer (GSI-61, Grason Stadler Inc., United States) was used to estimate hearing thresholds using TDH-50 $\mathrm{P}$ earphones and a Radioear B-71 bone vibrator. Calibrated GSI Tympstar middle ear analyzer was used to screen middle ear function. DPOAEs were recorded using an Echoport ILO292 (Otodynamics Ltd, United Kingdom) using v.6 software. A sound-treated room with permissible background noise level ${ }^{15}$ was utilized to carry out all the tests, including the baseline evaluations. Pure tone average was calculated using thresholds at $500 \mathrm{~Hz}, 1 \mathrm{kHz}$, and $2 \mathrm{kHz}$. cVEMP testing was done using the IHS SmartEP system. All the participants were instructed to sit upright in a comfortable chair. Commercially available abrasive gel Nuprep was used to clean the electrode sites and obtain acceptable impedance. The gold-plated cup electrodes were placed using Ten20 conductive paste and secured with surgical tape. 
The electrode montage used was as follows: noninverting electrode was placed on the upper one-third of the sternocleidomastoid muscle, inverting electrode was placed on the sternoclavicular junction, and the ground electrode was placed on the forehead. Absolute and interelectrode impedance were 5 and 2 Ohms, respectively. All participants were requested to turn their heads toward the opposite side of the ear being stimulated to obtain tension in the sternocleidomastoid muscle. A visual feedback monitor was used to maintain neck tension. The stimulus and analysis parameters were as follows: Tone bursts of $500 \mathrm{~Hz}$ with alternating polarity, ramped with rise/fall time and $1 \mathrm{~ms}$ plateau, were used. The stimulus intensity was $100 \mathrm{~dB}$ normal hearing level, with a repetition rate of 5.1/s. The waveform was analyzed using an analysis window of $74 \mathrm{~ms}$, with $20 \mathrm{~ms}$ prestimulus baseline. Responses were averaged across 256 sweeps after being bandpass filtered between $10 \mathrm{~Hz}$ and $1,500 \mathrm{~Hz}$ with an amplification of 5,000.

\section{Statistical Analysis}

Analysis was done using IBM SPSS software (version 23). Descriptive statistics such as mean and standard deviation were obtained for pure tone thresholds data, and cVEMP latencies and absolute amplitudes were analyzed using an independent sample $t$-test for between and within-group comparisons.

\section{Results}

The current study aimed to understand whether exposure to music from the violin influences an individual's saccular responses. The age range of all the participants was 21 to 27 years. The nonmusicians group had six participants (four male and two female) and the violinists' group had six participants of which one was male and five were females. Participants in the group of violinists had an average daily exposure of about one and a half hours and had been practicing the instrument for 5 years or more.

Mean values of pure tone average at all frequencies for non musicians and violinist group are reported in - Table $\mathbf{1}$.
Pure tone audiometry revealed hearing thresholds at all frequencies within the range of $25 \mathrm{~dB}$ hearing level suggesting hearing sensitivity within normal limits ${ }^{16}$ in both ears for both groups. However, hearing thresholds in the group of violinists were elevated in comparison to the nonmusician group, especially in the left ear as compared to the right ear, respectively.

Paired $t$-test was used to compare within and between-group cVEMP latencies and absolute amplitudes in the nonmusician and violinist group. Mean, standard deviation, and $p$-value are reported in - Table 2 .

Greater differences in latencies of both $\mathrm{P} 13$ and N23 peaks were observed in the violinist group. An average mean difference of $2 \mathrm{~ms}$ was seen for both the peaks in the violinist group; however, this difference was not statistically significant. Absolute amplitude comparison between the two groups also revealed larger absolute amplitude variations for P13 and N23 peaks, greater in the left ear as compared with the right, in the violinist group. In the right ear, the absolute amplitude of P13 was greater by $7 \mathrm{mV}$ as compared with the nonmusician group. In the left ear, the average mean difference for absolute amplitude was $4 \mathrm{mV}$, in comparison to the nonmusician group. Overall, both latency and absolute amplitude of P13 and N23 showed larger deviations in the violinist group (left ear > right ear); however, this difference was not statistically significant.

A within-group comparison between the ears of the violinists was performed. Mean, standard deviation, and $p$-value are reported in - Table $\mathbf{3}$.

Latencies of both peaks were greater in the left ear in comparison with the right ear, as mentioned previously. Similarly, the absolute amplitude of both peaks was greater in the left ear in comparison to the right; however, both latency and absolute amplitude differences were not statistically significant.

\section{Discussion}

This study aimed to understand whether exposure to music from the violin influences an individual's saccular responses. In our study, the violinist group had elevated cVEMP absolute

Table 1 Mean values of PTA for nonmusician and violinist group

\begin{tabular}{|l|l|l|l|l|}
\hline & \multicolumn{3}{|c|}{ Nonmusician } & \multicolumn{2}{c|}{ Violinist } \\
\hline Frequencies & \multicolumn{4}{|c|}{ PTA (dBHL) } \\
\hline & Right ear & Left ear & Right ear & Left ear \\
\hline $250 \mathrm{~Hz}$ & 14.16 & 11.6 & 17.5 & 21.6 \\
\hline $500 \mathrm{~Hz}$ & 10.8 & 10 & 15.8 & 19.16 \\
\hline $1 \mathrm{kHz}$ & 10 & 9.16 & 12.5 & 16.6 \\
\hline $2 \mathrm{kHz}$ & 9.16 & 10 & 10.8 & 14.16 \\
\hline $4 \mathrm{kHz}$ & 8.3 & 6 & 11.6 & 13.3 \\
\hline $8 \mathrm{kHz}$ & 6.6 & 6 & 13.3 & 12.5 \\
\hline PTA & 10 & 9.72 & 13.05 & 16.6 \\
\hline
\end{tabular}

Abbreviations: $\mathrm{dBHL}$, decibel hearing level; PTA, pure tone audiometry. 
Table 2 Mean, SD, and $p$-value of cVEMP latencies and absolute amplitude between nonmusician and violinist group

\begin{tabular}{|l|l|l|l|l|l|l|l|}
\hline \multicolumn{2}{|c|}{} & \multicolumn{2}{|c|}{ Nonmusician group $(\boldsymbol{n}=6)$} & \multicolumn{2}{c|}{ Violinist group $(\boldsymbol{n}=6)$} & \multicolumn{2}{c|}{$p$-Value } \\
\cline { 2 - 9 } & Right ear & Left ear & Right ear & Left ear & Right ear & Left ear \\
\hline \multirow{2}{*}{ Latencies $(\mathrm{ms})$} & $\mathrm{P} 13$ & $15.30 \pm 1.72$ & $14.23 \pm 1.19$ & $17.75 \pm 4.35$ & $16.66 \pm 2.76$ & 0.26 & 0.10 \\
\cline { 2 - 9 } & $\mathrm{N} 23$ & $22.36 \pm 0.4$ & $21.85 \pm 1.36$ & $22.83 \pm 2.63$ & $23.95 \pm 1.01$ & 0.70 & 0.08 \\
\hline $\begin{array}{l}\text { Absolute } \\
\text { amplitudes } \\
(\mu \mathrm{V})\end{array}$ & $\mathrm{P} 13$ & $44.09 \pm 10.43$ & $53.21 \pm 26.12$ & $51.74 \pm 27.49$ & $60.27 \pm 10.89$ & 0.57 & 0.58 \\
\cline { 2 - 8 } & $\mathrm{N} 23$ & $30.49 \pm 12.8$ & $37.39 \pm 19.90$ & $38.55 \pm 24.53$ & $41.75 \pm 12.24$ & 0.53 & 0.68 \\
\hline
\end{tabular}

Abbreviations: cVEMP, cervical vestibular evoked myogenic potential; SD, standard deviation.

Table 3 Mean, SD, and $p$-value of cVEMP latencies and absolute amplitudes between right and left ears in violinist group

\begin{tabular}{|l|l|l|l|l|l|}
\hline \multicolumn{2}{|c|}{} & \multicolumn{2}{c|}{ Violinist group ears $(\boldsymbol{n}=12)$} & \multicolumn{2}{c|}{$p$-Value } \\
\cline { 2 - 7 } \multicolumn{2}{|c|}{} & Right ear & Left ear & Right ear & Left ear \\
\hline \multirow{2}{*}{ Latencies (ms) } & $\mathrm{P} 13$ & $15.30 \pm 1.89$ & $16.6 \pm 3.02$ & 0.07 & 0.35 \\
\cline { 2 - 7 } & $\mathrm{N} 23$ & $22.36 \pm 0.53$ & $23.95 \pm 2.20$ & 0.36 & 0.08 \\
\hline $\begin{array}{l}\text { Absolute amplitudes } \\
(\mu \mathrm{V})\end{array}$ & $\mathrm{P} 13$ & $44.09 \pm 11.43$ & $60.27 \pm 11.93$ & 1.89 & 2.36 \\
\cline { 2 - 7 } & $\mathrm{N} 23$ & $30.49 \pm 14.10$ & $41.75 \pm 13.40$ & 0.83 & 0.96 \\
\hline
\end{tabular}

Abbreviations: cVEMP, cervical vestibular evoked myogenic potential; SD, standard deviation.

amplitudes and latencies. cVEMP latencies in the violinist group were prolonged by $2 \mathrm{~ms}$ in both ears and the absolute amplitudes were increased by 4 and $7 \mu \mathrm{V}$ in the right and left ear, respectively. This may be indicative of early changes occurring in the saccule ${ }^{17}$ due to continuous noise exposure, but the changes may not be significant as they are not involving the inferior vestibular nerve, hence maintaining the function of the sacculocollic pathway. ${ }^{18}$

Occupational noise exposure is often said to result in reduced excitability of the saccule. ${ }^{19,20}$ The group of violinists in our study had been exposed to violin music, on an average of one and a half hours, every day for more than 5 years. This type of continuous noise exposure of longer durations has proven to have detrimental effects ${ }^{21}$ on hearing, but these small changes in latencies and absolute amplitudes of cVEMP may reflect changes in the saccule that occur without any alterations in the auditory sensitivity. ${ }^{9}$ The similarity in the structure of the hair cells in the cochlea and vestibule and common blood supply of the cochlea ${ }^{22}$ and vestibular end-organs by the same artery support the likelihood of saccular deterioration related to cochlear damaging factors, ${ }^{23}$ resulting in slightly longer latencies in violinists. Studies also state that pathologies affecting the saccule, such as Meniere's disease are, likely to not affect the latencies of cVEMP, ${ }^{24}$ suggesting that the changes occurring are limited to the saccule.

Furthermore, larger absolute amplitudes of the P13 and N23 peaks CVEMP were seen in this group indicating the recruitment of the saccular receptors. Recruitment in these cells may be the result of bone-conducted vibrations that lead to irregular otolithic motion showing activation of neurons at higher stimulus levels showing a steep increase in firing with an increase in intensity. ${ }^{25}$ Long-term exposure to violin music may result in early damage at the morphological level, causing abnormal growth of saccular responses.

\section{Conclusion}

cVEMP may be used as a part of the auditory vestibular test battery to detect these prior changes. Detecting these early changes could help monitor them over a while and benefit cochlear and vestibular assessment and management. Future studies could benefit by incorporating a larger sample size and understanding the effect of age and duration of practice in early detection of these changes in musicians and accordingly provide remediation measures to avoid further deterioration in the system.

\section{Conflict of Interest}

None declared.

\section{Acknowledgments}

We thank all the participants for their contribution to this study.

\section{References}

1 Chasin M. Musicians and the prevention of hearing loss. In: Audio Engineering Society Conference: 2018 AES International Conference on Music Induced Hearing Disorders. Audio Engineering Society; 2018

2 Reybrouck M, Podlipniak P, Welch D. Music and noise: same or different? what our body tells us. Front Psychol 2019;10:115310.3389/fpsyg.2019.01153

3 Moussavi-Najarkola SA, Khavanin A, Mirzaei R, Salehnia M, Muhammadnejad A, Akbari M. Noise-induced outer hair cells' dysfunction and cochlear damage in rabbits. Iran Red Crescent Med J 2012;14(10):647-656 https://pubmed.ncbi.nlm.nih. gov/23285417

4 Le TN, Straatman LV, Lea J, Westerberg B. Current insights in noise-induced hearing loss: a literature review of the underlying mechanism, pathophysiology, asymmetry, and management options. J Otolaryngol Head Neck Surg 2017;46(1):4110.1186/s40463-017-0219-x

5 Mangabeira-Albernaz PL, Covell WP, Eldredge DH. Changes in the vestibular labyrinth with intense sound. Laryngoscope 1959;69:1478-1493 
6 Manabe Y, Kurokawa T, Saito T, Saito H. Vestibular dysfunction in noise induced hearing loss. Acta Otolaryngol Suppl 1995;519:262-264

7 Basner M, Babisch W, Davis A, et al. Auditory and non-auditory effects of noise on health. Lancet 2014;383(9925) :1325-1332

8 Raghunath G, Suting LB, Maruthy S. Vestibular symptoms in factory workers subjected to noise for a long period. Int J Occup Environ Med 2012;3(3):136-144

9 Oosterveld WJ, Polman AR, Schoonheyt J. Noise-induced hearing loss and vestibular dysfunction. Aviat Space Environ Med 1980;51(8):823-826

10 Ogido R, Costa EA, Machado Hda C. [Prevalence of auditory and vestibular symptoms among workers exposed to occupational noise]. Rev Saude Publica 2009;43(2):377-380

11 Singh NK, Sasidharan CS. Effect of personal music system use on sacculocollic reflex assessed by cervical vestibular-evoked myogenic potential: a preliminary investigation. Noise Health 2016;18(81):104-112

12 Emami SF. Acoustic sensitivity of the saccule and daf music. Iran J Otorhinolaryngol 2014;26(75):105-110

13 McBride D, Gill F, Proops D, Harrington M, Gardiner K, Attwell C. Noise and the classical musician. BMJ 1992;305(6868) :1561-1563

14 Rensing N, Schemmann H, Zalpour C. Musculoskeletal demands in violin and viola playing: a literature review. Med Probl Perform Art 2018;33(4):265-274

15 1-1999 AS. Maximum permissible ambient noise levels for audiometric test rooms. New York Acoust Soc Am. Published online 1999

16 Olusanya BO, Davis AC, Hoffman HJ. Hearing loss grades and the International Classification of Functioning, Disability and Health. Bull World Health Organ 2019;97(10):725-728

17 Stewart CE, Holt AG, Altschuler RA, et al. Effects of noise exposure on the vestibular system: a systematic review.
Front Neurol 2020;11:593919, https://www.frontiersin. org/article/10.3389/fneur.2020.593919

18 Murofushi T, Matsuzaki M, Mizuno M. Vestibular evoked myogenic potentials in patients with acoustic neuromas. Arch Otolaryngol Head Neck Surg 1998;124(5):509-512

19 Madappa M, Mamatha NM. Vestibular Evoked Myogenic Potential (VEMP) in Individuals with Noise Induced Hearing Loss (NIHL). PART-A Audiol. Published online 2010:109. Accessed on August 14 at: https://www.noiseandhealth. org/article. asp? issn=1463-1741; year=2010; volume =12; issue $=48$; spage $=191$; epage $=194$; aulast $=$ Kumar

20 Akin FW, Murnane OD, Proffitt TM. The effects of click and tone-burst stimulus parameters on the vestibular evoked myogenic potential (VEMP. J Am Acad Audiol 2003;14(9):500-509, quiz 534-535

21 Lie A, Skogstad M, Johannessen HA, et al. Occupational noise exposure and hearing: a systematic review. Int Arch Occup Environ Health 2016;89(3):351-372

22 Shin S-A, Lyu A-R, Jeong S-H, Kim TH, Park MJ, Park Y-H. Acoustic trauma modulates cochlear blood flow and vasoactive factors in a rodent model of noise-induced hearing loss. Int J Mol Sci 2019;20(21):531610.3390/ijms20215316

23 Sazgar AA, Dortaj V, Akrami K. Akrami S, Karimi Yazdi AR. Saccular damage in patients with high-frequency sensorineural hearing loss. Eur Arch Otorhinolaryngol 2006;263(7):608-613

24 Osei-Lah V, Ceranic B, Luxon LM. Clinical value of tone burst vestibular evoked myogenic potentials at threshold in acute and stable Ménière's disease. J Laryngol Otol 2008;122(5):452-457

25 Curthoys IS, Vulovic V. Vestibular primary afferent responses to sound and vibration in the guinea pig. Exp Brain Res 2011;210(3-4):347-352 